OPEN ACCESS

Edited by:

Wenbin Guo,

Central South University, China

Reviewed by:

Katharina Wenger,

University Hospital Frankfurt, Germany

Mark Mikkelsen,

Weill Cornell Medicine, United States

${ }^{*}$ Correspondence:

Zahra Shams

z.shams@umcutrecht.nI

Specialty section

This article was submitted to Applied Neuroimaging, a section of the journal

Frontiers in Neurology

Received: 31 May 2021

Accepted: 05 August 2021

Published: 07 September 2021

Citation:

Shams Z, van der Kemp WJM, Emir U, Dankbaar JW, Snijders TJ, de Vos FYF, Klomp DWJ, Wijnen JP and Wiegers EC (2021) Comparison of 2-Hydroxyglutarate Detection With SLASER and MEGA-SLASER at $7 T$.

Front. Neurol. 12:718423

do: 10.3389/fneur.2021.718423

\section{Comparison of 2-Hydroxyglutarate Detection With sLASER and MEGA-sLASER at 7T}

\author{
Zahra Shams ${ }^{1 *}$, Wybe J. M. van der Kemp ${ }^{1}$, Uzay Emir ${ }^{2}$, Jan Willem Dankbaar ${ }^{1}$, \\ Tom J. Snijders ${ }^{3}$, Filip Y. F. de Vos ${ }^{4}$, Dennis W. J. Klomp ${ }^{1}$, Jannie P. Wijnen ${ }^{1}$ and \\ Evita C. Wiegers ${ }^{1}$
}

${ }^{1}$ Department of Radiology, University Medical Center Utrecht, Utrecht, Netherlands, ${ }^{2}$ School of Health Sciences, Purdue University, West Lafayette, IN, United States, ${ }^{3}$ Department of Neurology \& Neurosurgery, University Medical Center Utrecht/UMC Utrecht Brain Center, Utrecht, Netherlands, ${ }^{4}$ Department of Medical Oncology, University Medical Center Utrecht, Utrecht, Netherlands

The onco-metabolite 2-hydroxyglutarate (2HG), a biomarker of IDH-mutant gliomas, can be detected with ${ }^{1} \mathrm{H}$ MR spectroscopy ( $\left.{ }^{1} \mathrm{H}-\mathrm{MRS}\right)$. Recent studies showed measurements of $2 \mathrm{HG}$ at $7 \mathrm{~T}$ with substantial gain in signal to noise ratio (SNR) and spectral resolution, offering higher specificity and sensitivity for $2 \mathrm{HG}$ detection. In this study, we assessed the sensitivity of semi-localized by adiabatic selective refocusing (SLASER) and J-difference MEsher-GArwood-semi-LASER (MEGA-sLASER) for 2HG detection at 7T. We performed spectral editing at long TE using a TE-optimized SLASER sequence $(110 \mathrm{~ms})$ and $\mathrm{J}$-difference spectroscopy using MEGA-SLASER (TE $=74 \mathrm{~ms})$ in phantoms with different $2 \mathrm{HG}$ concentrations to assess the sensitivity of $2 \mathrm{HG}$ detection. The robustness of the methods against $B_{0}$ inhomogeneity was investigated. Moreover, the performance of these two techniques was evaluated in four patients with IDH1-mutated glioma. In contrary to MEGA-sLASER, sLASER was able to detect $2 \mathrm{HG}$ concentration as low as $0.5 \mathrm{mM}$. In case of a composite phantom containing $2 \mathrm{HG}$ with overlapping metabolites, MEGA-sLASER provided a clean 2HG signal with higher fitting reliability (lower \%CRLB). The results demonstrate that SLASER is more robust against field inhomogeneities and experimental or motion-related artifacts which promotes to adopt sLASER in clinical implementations.

Keywords: 2-hydroxyglutarate, ultra-high field, SV MRS, MEGA-sLASER, sLASER, J-difference editing

\section{INTRODUCTION}

Mutations in IDH1 and IDH2 encoding genes result in the accumulation of 2-hydroxyglutarate (2HG) in glial brain tumor cells $(1,2)$. 2HG can therefore serve as a biomarker for IDH mutated gliomas (3). IDH mutations are highly diagnostic for diffuse gliomas and form an integral part of the contemporary WHO classification of brain tumors (4). It has been shown that patients with IDH1 and IDH2 mutated gliomas have better survival rates than patients with IDH wild type tumors $(1,5)$. Recent studies demonstrated that IDH mutations in glioma predict the response to chemotherapy in anaplastic glioma (6). Hence, the detection of 2HG in IDH-mutated gliomas can aid in the diagnosis and treatment planning of glioma patients. This can be achieved non-invasively with MR spectroscopy (MRS), provided that dedicated sequences are being used. 
The signal of $2 \mathrm{HG}$ at $2.25 \mathrm{ppm}(\mathrm{H} \gamma)$ in the $\mathrm{MR}$ spectrum overlaps with signals of glutamate, glutamine and $\gamma$ aminobutyric acid (GABA). Consequently, the specificity of $2 \mathrm{HG}$ detection is low when using MRS sequences with a short echo time $(7,8)$. To mitigate this problem, spectral editing at long echo time and J-difference spectroscopy became of interest $(9,10)$. The pioneers of these approaches at 3T were Choi et al. They optimized the point-resolved spectroscopy (PRESS) sequence for 2HG detection at an echo time (TE) of $97 \mathrm{~ms}$ (9), with reasonable sensitivity and specificity. To overcome overlapping of metabolite signals in the frequency range of $2 \mathrm{HG}$ 's $\mathrm{H} \gamma$ signal, MEsherGArwood-PRESS (MEGA-PRESS) sequence (TE $=68 \mathrm{~ms}$ ) was introduced. This sequence detects the $\mathrm{H}_{\alpha}$ signal at $4.02 \mathrm{ppm}$ with a more accurate quantification of $2 \mathrm{HG}$ levels compared to PRESS $97 \mathrm{~ms}$ (11). Andronesi et al. (10) designed a J-difference editing sequence specific to $2 \mathrm{HG}$ detection $(T E=75 \mathrm{~ms}$ ), based on the LASER (localization by adiabatic selective refocusing) technique to alleviate the chemical shift displacement error compared to the PRESS sequence.

Increased spectral resolution at ultra-high field strengths $(\geq 7 \mathrm{~T})$ allows a more reliable detection of overlapping metabolite signals such as Glutamate (Glu) and Glutamine (Gln) (12). Together with the increased signal to noise ratio at ultra-high field strengths, subtle changes in metabolite levels can be detected which leads to highly improved precision and quantification accuracy (13). This can be beneficial for $2 \mathrm{HG}$ detection, since $2 \mathrm{HG}$ levels can be lower than $5 \mathrm{mM}$ in tumor tissue $(8,11,14)$. At 7T, long echo time $(110 \mathrm{~ms})$ semi-localized by adiabatic selective refocusing (sLASER) sequence for $2 \mathrm{HG}$ detection was proposed and proved to outperform its PRESS counterpart because of less chemical shift displacement error in addition to less sensitivity to $\mathrm{B}_{1}^{+}$inhomogeneities $(15,16)$. Based on quantum-mechanical simulations, the optimal echo time and inter-pulse delays in this scheme were obtained, which resulted in a fully absorptive negative lineshape for $2 \mathrm{HG}$ at $2.25 \mathrm{ppm}$. This substantially improved discrimination between J-coupled spin resonances of 2HG and Glu, Gln, Glutathione (GSH), and GABA compared to long-TE (110 ms) sLASER at 3T (17). Moreover, 2HG-optimized echo time of $110 \mathrm{~ms}$ resulted in significantly higher $\mathrm{H} \gamma$ signal at $2.25 \mathrm{ppm}$ in comparison to data acquired with a short TE of 36 ms (15).

A "cleaner" signal for $2 \mathrm{HG}$ may be obtained when using Jdifference editing, even more at ultra-high field as the chemical shift dispersion is larger, and consequently co-editing of other metabolites is reduced. Also, much narrower spectral selectivity can be obtained with the same editing pulse bandwidth as used at $3 \mathrm{~T}$. Utilizing the unique resonance at $4.02 \mathrm{ppm}$ of $2 \mathrm{HG}$ in J-difference editing could make this technique very robust and specific for $2 \mathrm{HG}$ detection. However, the main drawback of performing editing at $7 \mathrm{~T}$ is that the water suppression is more challenging, which may affect the $2 \mathrm{HG}$ resonance at $4.02 \mathrm{ppm}$.

So far, long echo time acquisitions have been used as the most robust method for $2 \mathrm{HG}$ detection at $7 \mathrm{~T}$, while the feasibility and performance of edited MRS remained unclear. In this study, we compare long-TE sLASER and J-difference editing MEGA-sLASER acquisitions for 2HG detections at 7T regarding robustness and reliability. Furthermore, the resulting spectra from four patients with IDH-mutant glial tumors are shown as practical examples of applying these methods at $7 \mathrm{~T}$.

\section{MATERIALS AND METHODS}

\section{Phantom Preparation}

The sensitivity of the two methods in $2 \mathrm{HG}$ detection was assessed in five spherical phantoms with a diameter of $4 \mathrm{~cm}$. Four phantoms contained 2HG (DL- $\alpha$-Hydroxyglutaric acid disodium salt, Sigma Aldrich) at different concentrations and glycine (Gly) as a reference peak where the concentrations of 2HG/Gly (mM/mM) were $0.5 / 5,1 / 10,1.6 / 10,5.8 / 10$. The fifth phantom contained $5 \mathrm{mM} 2 \mathrm{HG}, 1 \mathrm{mM}$ gamma-aminobutyric acid (GABA), $5 \mathrm{mM}$ glutamate (Glu), $5 \mathrm{mM}$ glutamine (Gln), $10 \mathrm{mM}$ creatine $(\mathrm{Cr}), 10 \mathrm{mM}$-acetyl aspartic acid (NAA), and $7 \mathrm{mM}$ myo-inositol (mIn). We added PBS (phosphate-buffered saline) solution to the phantoms as a buffer solution. The $\mathrm{pH}$ was adjusted to 7.2 by adding small drops of sodium hydroxide whilst measuring $\mathrm{pH}$ with a $\mathrm{pH}$-meter (SevenCompact S210 from Mettler Toledo).

\section{Acquisitions}

MR experiments were performed on a 7T MR scanner (Philips, Achieva, Best, NL) equipped with a 32-channel receive-only and 8-channel transmit coil (Nova Medical, Inc., Burlington, MA, USA). We performed sLASER measurements at TE $=110 \mathrm{~ms}$ and the delays between the frequency offset corrected inversion (FOCI) refocusing pulses were tuned as in Emir et al. (15) to achieve optimized J-coupling patterns for 2HG (Figure 1A). Spectral editing with MEGA-sLASER, with a TE $=74 \mathrm{~ms}$ adapted from Andreychenko et al. (18), was performed using two $180^{\circ}$ frequency selective Gaussian pulses with a bandwidth of $88 \mathrm{~Hz}$ which were tuned symmetrically from the water resonance at 1.9 and $7.5 \mathrm{ppm}$, in the odd and even acquisitions, respectively (Figure 1B).

\section{Phantom Experiments}

The offset frequencies of the Gaussian pulses were shifted 0.12 ppm from the patient values because of the lower temperature of the phantom (room temperature). The carrier frequency of the FOCI pulses for sLASER and MEGA-sLASER were 2.7 and $3.0 \mathrm{ppm}$, respectively. The following acquisition parameters were kept the same for all experiments: number of signal averages (NSAs) was 128 for all phantoms and 256 for the one with lowest concentration of $2 \mathrm{HG}$. TR $(5 \mathrm{~s})$; spectral width $(6 \mathrm{kHz})$; voxel size $\left(8 \mathrm{~cm}^{3}\right.$ isotropic); transverse voxel orientation, VAPOR water suppression (19); 16 steps of phase cycling; and number of complex points (np) 2048. $\mathrm{B}_{0}$ was optimized for the selected volumes by FASTMAP (20) with up to 2 nd order shim terms (PB-2nd) using the MRS voxel as a target volume. In additional experiments, we assessed the performance of the two methods in less optimal shimming conditions, as we expected in invivo experiments. The local $\mathrm{B}_{0}$ field in the voxel was perturbed 


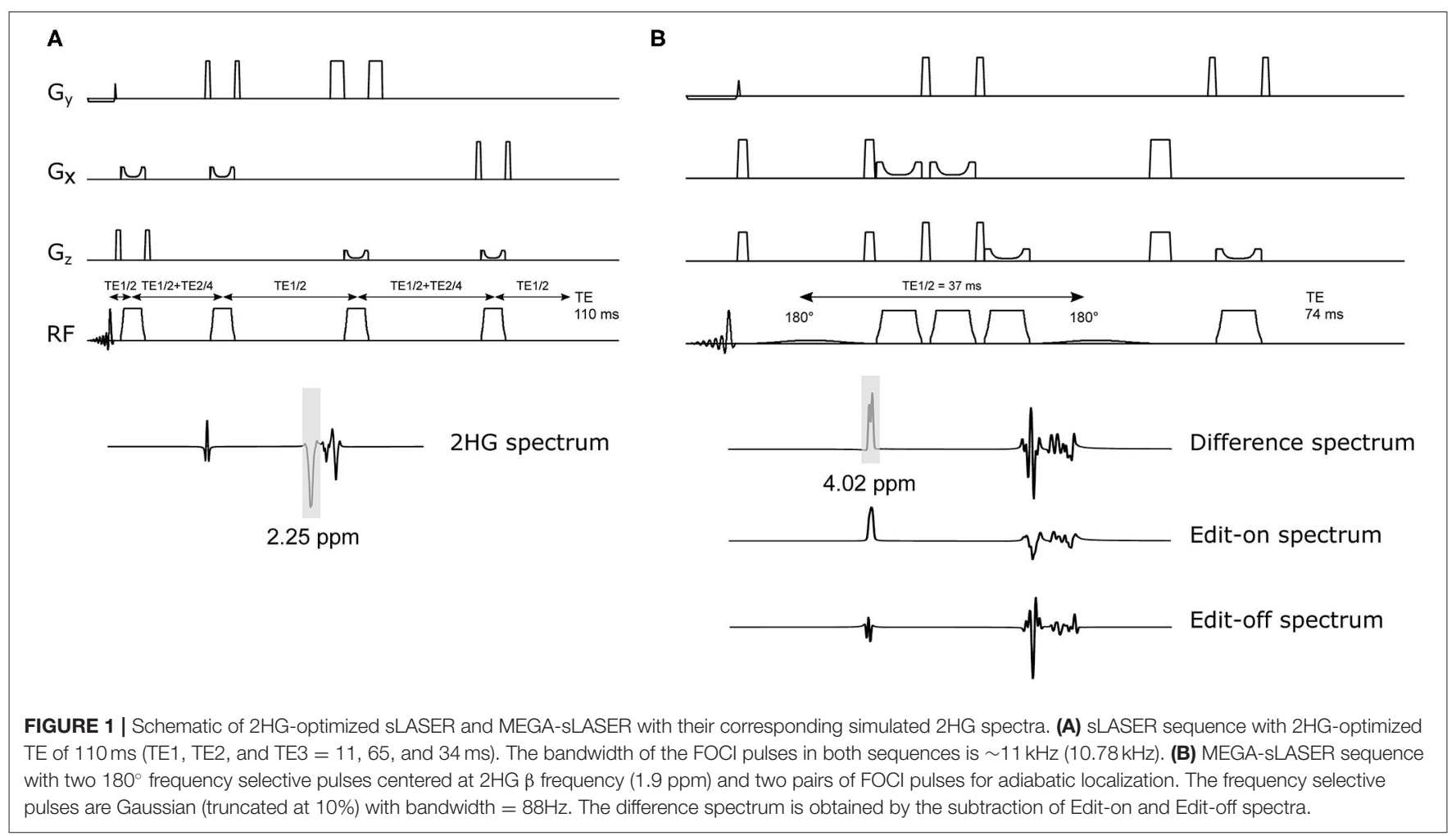

by changing the $\mathrm{z}$ component of the shim gradients up to $0.045 \mathrm{mT} / \mathrm{m}$.

\section{Patient Study}

The study was approved by the local ethical committee. We obtained informed consent from four patients with IDH1mutated glioma who then participated in this study: patient 1 and 2 with diffuse astrocytoma, WHO grade II, patient 3 with anaplastic astrocytoma, WHO grade III, and patient 4 with diffuse astrocytoma, WHO grade II, that developed to grade IV at time of progression. All IDH-mutations were confirmed by a certified neuropathologist by means of next-generation sequencing. These patients had received different types of treatments i.e., chemotherapy, proton-beam irradiation and photon radiotherapy. An overview of clinical characteristics, including exact IDH1-mutation as well as previous and ongoing treatments at time of the MRS are given in Supplementary Table 1. The location of the MRS voxel within the tumor was appointed by an experienced neuroradiologist (JWD) on a T2-weighted FLAIR image (3D TSE sequence with $1 \mathrm{~mm}$ isotropic resolution). MRS measurements were performed with the same acquisition parameters as the phantom study. Patient 4 had titanium surgical material in his skull, therefore we had to lengthen the TR to $6 \mathrm{~s}$ to remain within SAR (specific absorption rate) limits. NSAs for sLASER and MEGA-sLASER were 64 and 128, and for patient 1 were 32 and 64, respectively. $2 \mathrm{HG}$ spectra were acquired from voxel sizes of $8 \mathrm{~cm}^{3}$ (patient 1), $9.6 \mathrm{~cm}^{3}$ (patient 2), $4.98 \mathrm{~cm}^{3}$ (patient 3), and $8 \mathrm{~cm}^{3}$ (patient 4).
No additional outer volume suppression (OVS) was used except for patient 1 .

\section{Data Processing and Analysis}

The data were coil combined (21), frequency and phase aligned with the FID-A toolbox (22). For MEGA-sLASER, the on and off spectra were subtracted after phase and frequency alignment followed by averaging. All the spectra were analyzed using LCModel (23). Spectral simulation was done using Vespa library of GAMMA (24) to create the basis sets for LCModel analysis. The concentration of $2 \mathrm{HG}$ in the $2 \mathrm{HG}$-Gly phantoms was estimated using the water spectrum as a reference. The water concentration was assumed to be $55.6 \mathrm{M}$ (bulk water) in the phantom. Estimated $2 \mathrm{HG}$ and Gly concentrations were corrected for T2 relaxation [T2 (Gly) $=1,500 \mathrm{~ms}$ and T2 (2HG) $=650 \mathrm{~ms}$ ] (16). The effect of $\mathrm{T} 1$ relaxation rate was not taken into account. The metabolites included in the basis set for the sLASER experiment were: 2-HG, alanine (Ala), ascorbate (Asc), aspartate (Asp), creatine (Cr), GABA, Gln, Glu, glycine (Gly), myo-Inositol, Lactate (Lac), N-acetylaspartylglutamate (NAAG), phosphocholine (PCho), phosphocreatine (PCr), phosphoethanolamine (PE), scyllo-inositol (sIns), taurine (Tau), glucose (Glc), glycerophosphocholine (GPC), and GSH. The basis set for MEGA-sLASER experiment included 2HG, GABA, Glu, Gln, GSH, NAA, and NAAG. We set the DKNTMN parameter of LCModel to 0.2 to make the spline baseline flexible enough to automatically account for highly variable macromolecule and lipid signals. For sLASER with long TE of $110 \mathrm{~ms}$, the macromolecules contribution can be neglected (25). 
For the 2HG-Gly phantoms, the edited spectra only contained signals from $2 \mathrm{HG}$. The relative $2 \mathrm{HG}$ and Gly concentration in these phantoms was calculated by deriving the concentration of Gly from the edit-off spectra (spectra with editing pulses tuned on $7.5 \mathrm{ppm}$ ).

\section{RESULTS}

Figure 2 shows the LCModel fits in 2HG-Gly phantoms with corresponding CRLBs for both sLASER and MEGA-sLASER acquisitions. Using J-difference editing, the multiplet at 4.02 ppm is resolved in the spectrum while removing the signal contribution from Gly after the subtraction of two subsequent acquisitions, with and without the frequency selective pulse. In both techniques, $2 \mathrm{HG}$ can be detected with a CRLB (\%) of $<20 \%$ except for the phantom with lowest $2 \mathrm{HG}$ concentration $[2 \mathrm{HG} / \mathrm{Gly}(\mathrm{mM})=0.5 / 5]$ where MEGA-sLASER failed according to the CRLB of the fit 59\%. Using sLASER, we were able to detect $2 \mathrm{HG}$ concentration of as low as $0.5 \mathrm{mM}(\mathrm{CRLB}=30 \%)$ with at least 32 number of signal averages. sLASER and MEGAsLASER were able to reproduce the real 2HG-Gly concentration ratio of $0.58(5.8 / 10 \mathrm{mM})$ in the corresponding phantom with the errors of 1.3 and $5.4 \%$, respectively. For the phantom with real 2HG-Gly relative concentration of $0.16(1.6 / 10 \mathrm{mM})$, the errors produced by sLASER and MEGA-sLASER were 1.28 and $0.6 \%$, respectively. The mean errors of the relative concentration for the other two phantoms were 9.9\% for sLASER and 26.3\% (2HG overestimation in both phantoms) for MEGA-sLASER. The $2 \mathrm{HG}$ to Gly ratio (After T2 correction) is plotted as a function of the number of signal averages in Figure 3. Only considering the
NSAs where the $2 \mathrm{HG}$ \%CRLB was lower than 30\%, MEGAsLASER resulted in overestimation of $2 \mathrm{HG} / \mathrm{Gly}$ in the phantom with $1 \mathrm{mM} 2 \mathrm{HG}$.

In the presence of other overlapping metabolites, MEGAsLASER resulted in more precise detection of $2 \mathrm{HG}$ with relative concentration $(/ \mathrm{NAA})$ of $0.43(\mathrm{CRLB}=6 \%)$ compared to 0.36 $(\mathrm{CRLB}=13 \%)$ with sLASER as shown in Figure 4 [the true relative concentration $(/ \mathrm{NAA})=0.5]$. In addition, the GABA CRLB was lower $(\mathrm{CRLB}=11 \%)$ with MEGA-sLASER compared to sLASER (CRLB $=16 \%$ ). Tables 1, 2 summarize the effect of metabolite signal overlap on CRLB (\%) and concentrations (/NAA) by removing the overlapping metabolites from the basis set. Excluding GABA from the basis set had negligible or even no influence on detecting $2 \mathrm{HG}$ using MEGA-sLASER, while the \%CRLB for $2 \mathrm{HG}$ decreased (from 13 to 5\%) after GABA's removal from the basis set in sLASER technique. Removing Glu and Gln from the basis set had almost no influence on quantification of $2 \mathrm{HG}$ using sLASER (Table 2). While the reliability of $2 \mathrm{HG}$ fit (\%CRLB) was affected by the linewidth in the sLASER sequence, the MEGA-sLASER sequence exhibited the same \%CRLB of the metabolites in MR spectra with increasing linewidth (Figure 5). The reported linewidth is the water linewidth reported by LCModel.

Figure 6 shows the spectra from the four patients that participated in this study. The choline linewidth for Patient 1, 2 , 3, and 4 were $29.13,11.49,12.45$, and $15.71 \mathrm{~Hz}$, respectively. For these patients, partial volume of the tumor in the MRS voxel was estimated by a neuroradiologist (JWD) as the percentage of the MRS voxel filled with the FLAIR abnormality that were 90, 90,80 , and $80 \%$ with estimated tumor likelihood (the possibility

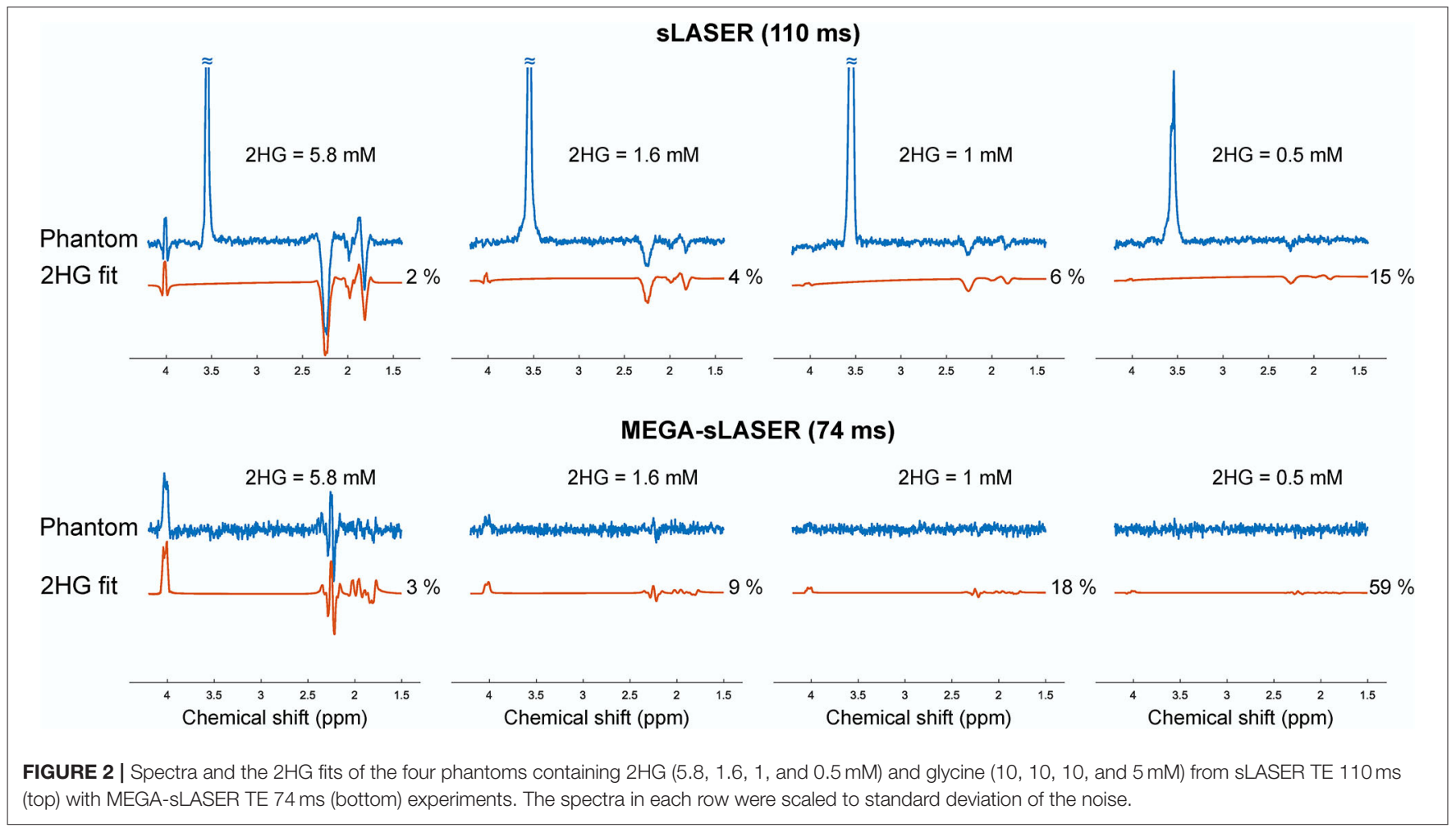



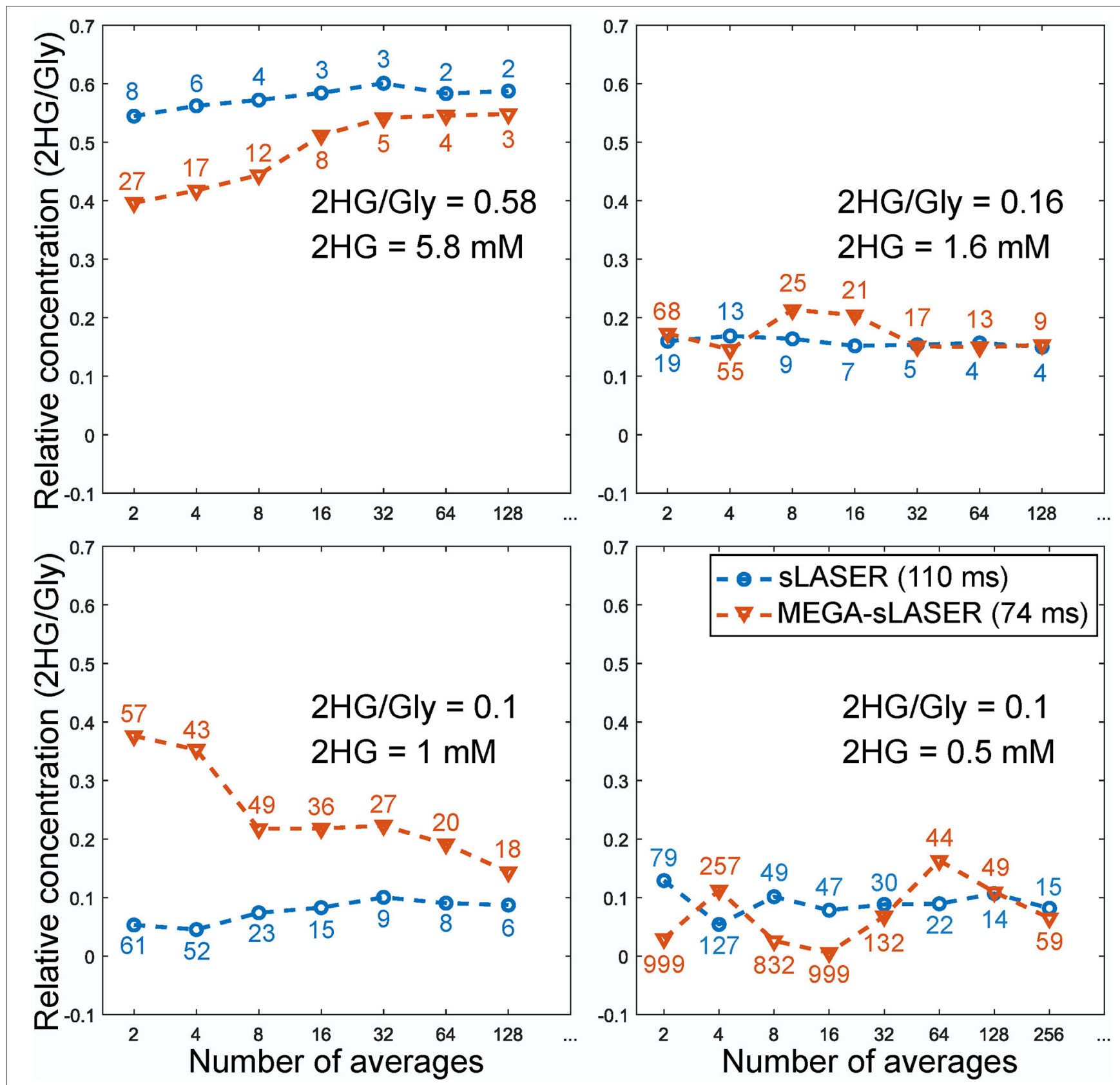

FIGURE 3 | Relative concentration of $2 \mathrm{HG}$ to glycine in the phantoms as a function of number of averages. The numbers correspond to the CRLBs of the $2 \mathrm{HG}$ fit. The concentration of Gly for MEGA-sLASER measurements was derived from the edit-off spectra.

of the abnormal tissue in the MRS voxel being tumorous) of $25,100,75$, and $50 \%$, respectively. In patient 1 , with low probability of the MRS voxel being filled with genuine tumor, $2 \mathrm{HG}$ was detected by sLASER $(\% \mathrm{CRLB}=27)$ while no $2 \mathrm{HG}$ was detected with MEGA-sLASER (\%CRLB = 159). In patient 2 with high tumor likelihood and high partial volume of tumor, both methods perform well in $2 \mathrm{HG}$ detection. By excluding 2HG from the sLASER basis set, a residual signal is seen at $2.25 \mathrm{ppm}$ from the fit although CRLB of GABA improved a bit. The peak at around $4.02 \mathrm{ppm}$ in the edited spectrum can be purely assigned to $2 \mathrm{HG}$. In patient 3 , the editing method failed. For the chosen voxel location, the spoilers were not strong enough, leading to large spurious echoes around the $2 \mathrm{HG}$ frequency at $4.02 \mathrm{ppm}$. When excluding $2 \mathrm{HG}$ from the basis set, the fit resulted in residuals at $2 \mathrm{HG}$ resonance frequency of 2.25 ppm (Supplementary Figure 1A). The correlation coefficients between 2HG and either GABA, Glu or Gln reported by LCModel for this case were $0.26,-0.2$, and 0.22 respectively, meaning 


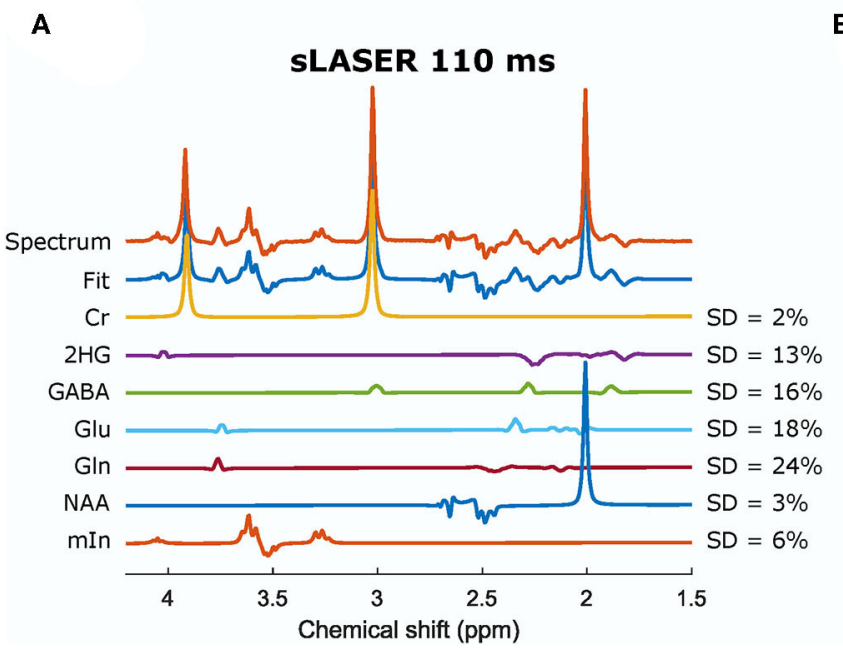

B

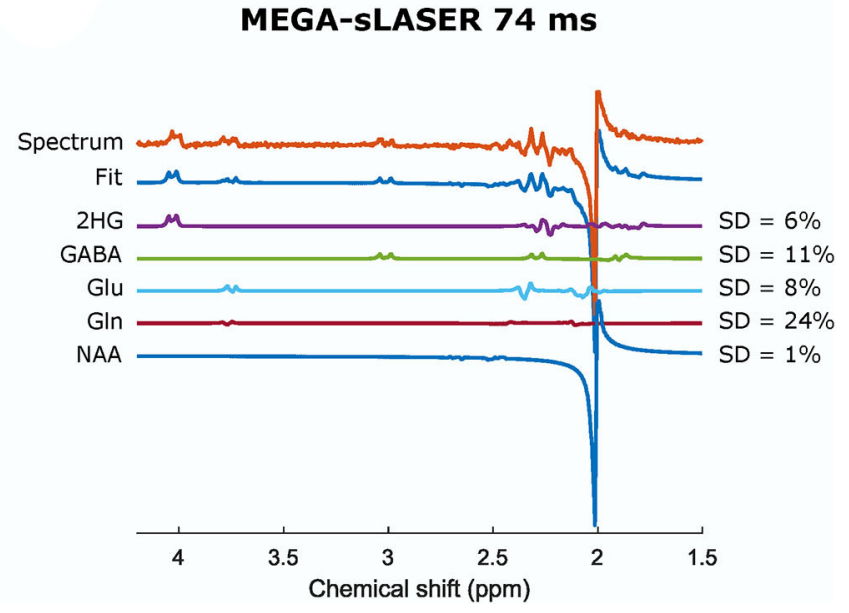

FIGURE 4 | Spectra and the fits from a phantom containing 2HG, Cr, NAA, Glu, Gln, GABA, and mln acquired from sLASER TE = 110 ms (A) and MEGA-sLASER TE $=74 \mathrm{~ms}$ (B). The relative concentrations (/NAA) can be found in Table 2. Cr, creatine; NAA, N-acetylaspartate; Glu, glutamate; Gln, glutamine; GABA, $\gamma$-aminobutyric acid; mln, myo-inositol.

TABLE 1 | CRLB (\%) of overlapping metabolites.

\begin{tabular}{llccccc}
\hline & \multicolumn{5}{c}{ CRLB (\%) } \\
\cline { 3 - 7 } & & 2HG & GABA & Glu+GIn & Glu & Gln \\
\hline \multirow{2}{*}{ Without 2HG } & MEGA-sLASER & & 13 & 12 & 10 & 32 \\
& SLASER & & 20 & 14 & 17 & 29 \\
Without GABA & MEGA-sLASER & 6 & & 8 & 8 & 22 \\
& SLASER & 5 & & 14 & 21 & 23 \\
Without Glu-GIn & MEGA-sLASER & 7 & 10 & & & \\
& SLASER & 14 & 18 & & & 24 \\
& MEGA-sLASER & 6 & 11 & 9 & 8 & 24 \\
& SLASER & 13 & 15 & 13 & 18 & 24 \\
\hline
\end{tabular}

Overlapping metabolites such as $2 \mathrm{HG}, \mathrm{GABA}$, Glu, and Gln were removed from the basis set to examine the influence on the resulting fit. Gray shaded areas represent the excluded metabolites.

that the 2HG signal did not exhibit significant correlation with those metabolites. In patient 4 no $2 \mathrm{HG}$ was detected with either MEGA-sLASER or sLASER. Again, by removing $2 \mathrm{HG}$ from the basis set, no considerable change in the residual at 2.25 can be observed (Supplementary Figure 1B).

\section{DISCUSSION AND CONCLUSION}

In this study, we compared sLASER and MEGA-sLASER for detecting $2 \mathrm{HG}$ at $7 \mathrm{~T}$ in vitro and in patients. The sensitivity of the two methods for $2 \mathrm{HG}$ detection was assessed with four phantoms containing $2 \mathrm{HG}$ at different concentrations ranging between 0.5 and $5.8 \mathrm{mM}$. At low SNR and low levels of $2 \mathrm{HG}$ (e.g., $1 \mathrm{mM}$ ), the $2 \mathrm{HG}$ concentration using MEGA-sLASER was overestimated (Figure 3). sLASER appeared to be more sensitive
TABLE 2 | The relative concentrations (/NAA) obtained from MEGA-sLASER and sLASER when excluding the overlapping metabolites from the basis set.

\begin{tabular}{|c|c|c|c|c|c|c|}
\hline & & \multicolumn{5}{|c|}{ Concentration (/NAA) } \\
\hline & & $2 \mathrm{HG}$ & GABA & Glu+Gln & Glu & Gln \\
\hline \multirow[t]{2}{*}{ Without 2HG } & MEGA-sLASER & & 0.09 & 0.42 & 0.28 & 0.15 \\
\hline & sLASER & & 0.13 & 0.72 & 0.41 & 0.31 \\
\hline \multirow[t]{2}{*}{ Without GABA } & MEGA-sLASER & 0.42 & & 0.52 & 0.34 & 0.18 \\
\hline & SLASER & 0.33 & & 0.73 & 0.33 & 0.4 \\
\hline \multirow[t]{2}{*}{ Without Glu-Gln } & MEGA-sLASER & 0.39 & 0.11 & & & \\
\hline & SLASER & 0.36 & 0.15 & & & \\
\hline \multirow[t]{2}{*}{ All metabolites } & MEGA-sLASER & 0.43 & 0.09 & 0.47 & 0.31 & 0.15 \\
\hline & SLASER & 0.36 & 0.16 & 0.73 & 0.37 & 0.36 \\
\hline
\end{tabular}

True concentrations are: $2 H G / N A A=0.5, G A B A / N A A=0.1$, Glu/NAA $=0.5$, Gln/NAA $=0.5$. Gray shaded areas represent the excluded metabolites.

to $2 \mathrm{HG}$ detection at lower concentrations $(0.5 \mathrm{mM}$ with CRLB of $15 \%$ ) where MEGA-sLASER failed in detecting $2 \mathrm{HG}$ according to the reported CRLB of 59\%. Also, according to $2 \mathrm{HG}$-Gly ratio, the LCModel fits of sLASER were more consistent across the number of NSAs. It could be that the LCModel fit of $2 \mathrm{HG}$ in the edited spectra is suboptimal because of the absence of a prominent Gly peak. This situation could occur in fitting of edited MR spectra of tumors with very low NAA levels where subtraction of the spectra leads to only $2 \mathrm{HG}$ signals with some other co-edited metabolites such as GABA and Glu. We showed an example of such a spectrum in Figure 6 (patient 2).

The effect of linewidth on $2 \mathrm{HG}$ detection was evaluated by gradually increasing the $\mathrm{z}$ component of the shim and analyzing the changes in CRLB of the $2 \mathrm{HG}$ fit. When the linewidth was larger (a change from 13.1 to $14.6 \mathrm{~Hz}$ ), almost all the CRLBs for 

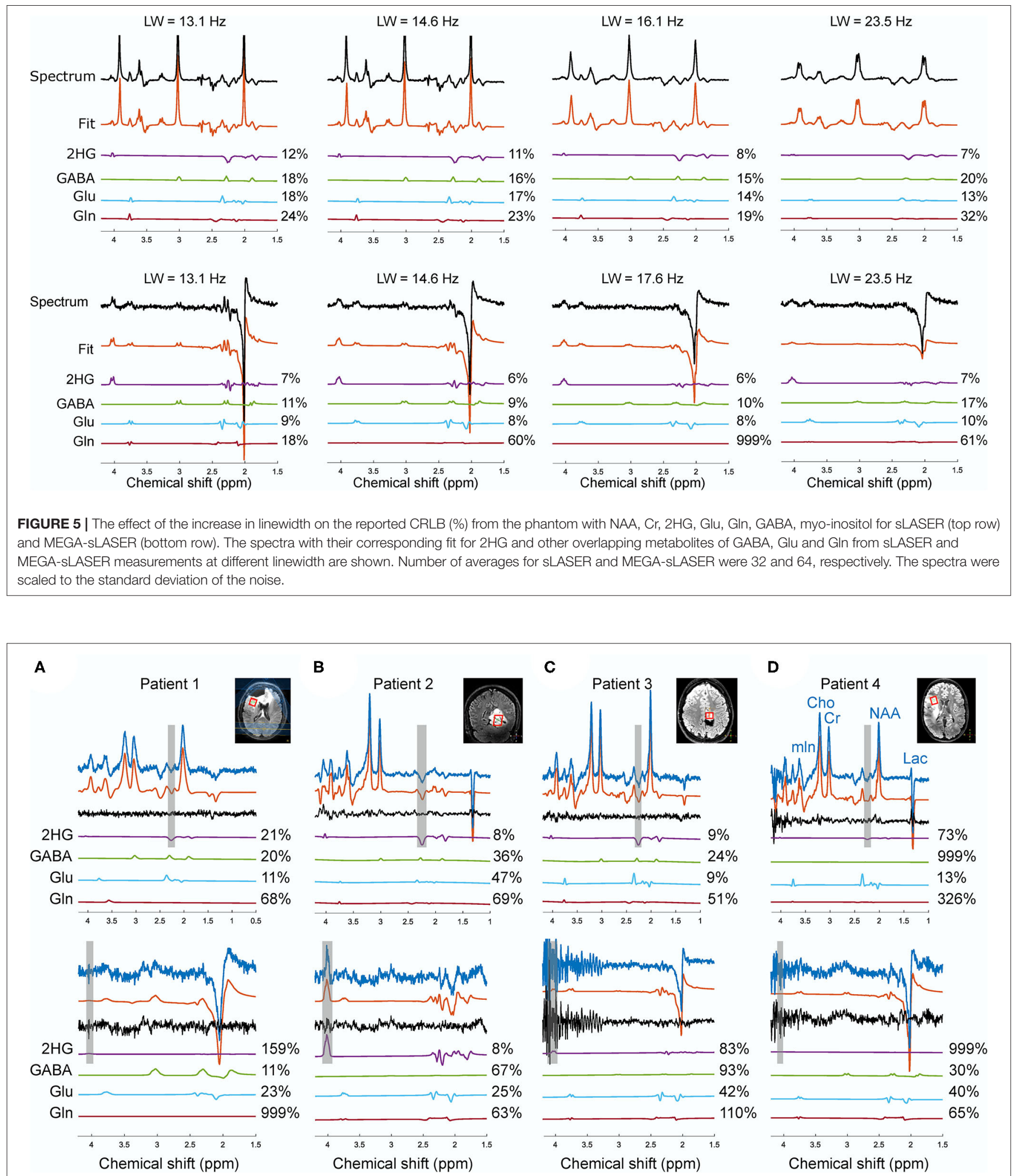

FIGURE 6 | The spectra from sLASER (upper spectra) and MEGA-SLASER (lower spectra) acquired in patients 1 (A), 2 (B), 3 (C), 4 (D) with histologically confirmed $\mathrm{IDH} 1$-mutated glioma. The frequency ranges of $2 \mathrm{HG} \gamma(2.25 \mathrm{ppm})$ and $\alpha(4.02 \mathrm{ppm})$ signals are shaded in gray. The voxel sizes for patients 1, 2, 3, and 4 were 8, 9.6 , 4.98 , and $8 \mathrm{~cm}^{3}$, respectively. 
2HG (12 and 11\%) and other overlapping metabolites remained the same in the sLASER method. But the linewidth of $16.1 \mathrm{~Hz}$ led to a decrease in $2 \mathrm{HG}$ CRLB (8\%) and a change in CRLBs of other metabolites. That means a linewidth of larger than $16 \mathrm{~Hz}$ will cause some signal stealing between $2 \mathrm{HG}$ and the overlapping metabolites in the sLASER sequence. On the other hand, broader linewidth did not have a noticeable impact on the fit reliability (\%CRLB) for the MEGA technique where the edited signal at 4.02 ppm is uniquely generated via the J-couplings of $2 \mathrm{HG}$. Despite the optimized interpulse delay aiming at a distinct lineshape for $2 \mathrm{HG}$, the remaining overlapping resonances from GABA, Glu and Gln, combined with poorer shim conditions in brain tumor tissue, may be more problematic than demonstrated here in phantoms. Nevertheless, in vivo MEGA-sLASER might be more sensitive to $\mathrm{B}_{0}$ inhomogenities for two reasons; firstly, narrowband frequency selective pulses tuned on specific frequencies would not perform optimally under strong inhomogeneous $\mathrm{B}_{0}$ conditions, which will consequently affect editing efficiency even though CRLB reports low numbers. Secondly, efficiency of water suppression will be also reduced, which ultimately leads to the $2 \mathrm{HG}$ resonance at $4.02 \mathrm{ppm}$ being obscured by water signal $(26,27)$. The acquisition in patient 1 , post-surgery and treated with chemotherapy, is an example of such a situation. The broad linewidth and its consequent neighboring signals contamination (2HG signal stealing from Glu and GABA) might be the reason for the low 2HG CRLB (27\%) for sLASER; the same as observed in the phantom with linewidth of $23 \mathrm{~Hz}$.

In addition to the phantom study, we showed some practical examples to assess the performance of these methods in patients. The four patients participated in this study had been under various treatments. Therefore, the presence and level of $2 \mathrm{HG}$ at the time of the scan was ambiguous. However, these cases demonstrate the practicality of each method for evaluating the performance of these two techniques in terms of robustness in patients. As discussed above, reliable detectability of $2 \mathrm{HG}$ depends on the linewidth. In our patient study, except for patient 1 , the quality of the $\mathrm{B}_{0}$ shimming was acceptable [according to Juchem et al. (28)] with the mean in vivo choline linewidth of $13.22 \pm 2.21 \mathrm{~Hz}$. In terms of spectral quality, sLASER was more robust across the patients with various voxel locations and sizes. For patient 2 (no surgery), both methods succeeded in detection of $2 \mathrm{HG}$. Most probably, suboptimal $\mathrm{B}_{0}$ shimming outside the voxel adjacent to the post-surgery cavity compromised water suppression and led to generation of spurious echoes in the MEGA-sLASER spectrum for patient 3 (Figure 6C).

All the patients had undergone surgery, either biopsy or subtotal resection, before their MR scan and had been receiving chemo- and/or radiotherapy when participating in this study. As this study did not focus on the effect of treatment, we did not evaluate the $2 \mathrm{HG}$ concentration in relation to treatment and therapy. Therefore, we compared the two methods based on the $2 \mathrm{HG}$ CRLB reported by LCModel rather than the $2 \mathrm{HG}$ levels.

To have comparable SNR for the sLASER and MEGAsLASER, the MEGA-editing measurements in patients were acquired with twice as many NSA and therefore a longer scan time for MEGA-sLASER. Being based on the subtraction of two spectra, editing techniques are susceptible to motion and scanner frequency drift which can result in underestimation of $2 \mathrm{HG}$ signal. Real-time motion and $\mathrm{B}_{0}$ correction may help to achieve more stability against motion and scanner-induced artifacts (29). In addition to a higher sensitivity of edited MRS to temporal frequency drift or $\mathrm{B}_{0}$ inhomogeneities, frequency selective Gaussian pulses used as editing pulses are sensitive to changes in $\mathrm{B}_{1}^{+}$field. Gaussian frequency selective pulses had a very narrow bandwidth which resulted in dependence of refocusing efficiency to $\mathrm{B}_{1}^{+}$(Supplementary Figure 2). It has been shown for GABA editing that the integrated signal of the edited multiplet decreased by a factor of approximately 15 when the $\mathrm{B}_{1}^{+}$is 70 or $130 \%$ compared to $100 \%$ (29), which could cause a false negative even with a low CRLB. Therefore, due to their $\mathrm{B}_{1}^{+}$insensitivity, asymmetric adiabatic editing pulses are favorable at 7T (29). However, adiabatic editing pulses will increase the RF deposition and therefore an even longer TR and acquisition time would be required.

Metabolic imaging of many more glioma-associated oncometabolites than $2 \mathrm{HG}$ has been explored recently $(30,31)$. Ultra high field provides robust molecular feature extraction in vivo (32) that facilitates high precision non-invasive clinical biomarker imaging for diagnosis in glioma patients. This suggests a need for an MRS sequence capable of simultaneous detection of those oncometabolites with high sensitivity and specificity, and thereby excluding the use of editing sequences. The 2HG-optimized sLASER $110 \mathrm{~ms}$ demonstrated both distinct cystathionine and $2 \mathrm{HG}$ peaks in oligodendroglioma patients with IDH-mutation and $1 \mathrm{p} / 19 \mathrm{q}$ codeletion at $7 \mathrm{~T}$ (33).

In conclusion, the patients' results showed that the MEGAediting method is less robust in cases of poor water suppression and $\mathrm{B} 0$ and $\mathrm{B} 1$ inhomogeneities than sLASER. Together with the finding that the fit reliability for $2 \mathrm{HG}$ in sLASER is similar to MEGA-sLASER promotes to choose for a sLASER implementation in clinical setting. Also, sLASER is a simpler implementation with the benefit of preserving signals from all other metabolites.

\section{DATA AVAILABILITY STATEMENT}

The raw data supporting the conclusions of this article will be made available by the authors upon personal request.

\section{ETHICS STATEMENT}

The studies involving human participants were reviewed and approved by the Medical Ethics Review Board (METC) from the University Medical Center Utrecht. The patients provided their written informed consent prior to participation in this study.

\section{AUTHOR CONTRIBUTIONS}

ZS, JW, EW, UE, JD, and DK designed the study. ZS and WK made the phantoms. ZS, EW, and JW conducted the experiments. ZS analyzed the data. ZS, JW, EW, and JD were involved in data 
interpretation. JD was responsible for patient recruitment and assigned the lesions and performed the radiological reading. TS and FV assisted in patient recruitment and reviewed clinical data. All authors drafted the manuscript and approved the final version of the manuscript.

\section{FUNDING}

This study was supported by funding from the H2020 program of the European Union; Eurostars Project E!12449 IMAGINE!.

\section{REFERENCES}

1. Yan H, Parsons DW, Jin G, McLendon R, Rasheed BA, Yuan W, et al. IDH1 and IDH2 Mutations in Gliomas. N Engl J Med. (2009) 360:765-73. doi: $10.1056 /$ nejmoa 0808710

2. Dang L, White DW, Gross S, Bennett BD, Bittinger MA, Driggers EM, et al. Cancer-associated IDH1 mutations produce 2-hydroxyglutarate. Nature. (2009) 462:739-44. doi: 10.1038/nature08617

3. Yen KE, Bittinger MA, Su SM, Fantin VR. Cancer-associated IDH mutations: biomarker and therapeutic opportunities. Oncogene. (2010) 29:6409-17. doi: 10.1038/onc.2010.444

4. Louis DN, Perry A, Reifenberger G, von Deimling A, Figarella-Branger D, Cavenee WK, et al. The 2016 World health organization classification of tumors of the central nervous system: a summary. Acta Neuropathol. (2016) 131:803-20. doi: 10.1007/s00401-016-1545-1

5. Parsons DW, Jones S, Zhang X, Lin JCH, Leary RJ, Angenendt P, et al. An integrated genomic analysis of human glioblastoma multiforme. Science. (2008) 321:1807-12. doi: 10.1126/science.1164382

6. van den Bent MJ, Tesileanu CMS, Wick W, Sanson M, Brandes AA, Clement $\mathrm{PM}$, et al. Adjuvant and concurrent temozolomide for $1 \mathrm{p} / 19 \mathrm{q}$ non-co-deleted anaplastic glioma (CATNON; EORTC study 26053-22054): second interim analysis of a randomised, open-label, phase 3 study. Lancet Oncol. (2021) 22:813-23. doi: 10.1016/s1470-2045(21)00090-5

7. Crisi G, Filice S, Michiara M, Crafa P, Lana S. 2-Hydroxyglutarate detection by short echo time magnetic resonance spectroscopy in routine imaging study of brain glioma at 3.0 T. J Comput Assist Tomogr. (2018) 42:469-74. doi: 10.1097/RCT.0000000000000705

8. Suh CH, Kim HS, Jung SC, Choi CG, Kim SJ. 2-Hydroxyglutarate MR spectroscopy for prediction of isocitrate dehydrogenase mutant glioma: a systemic review and meta-analysis using individual patient data. Neuro Oncol. (2018) 20:1573-83. doi: 10.1093/neuonc/noy113

9. Choi C, Ganji SK, DeBerardinis RJ, Hatanpaa KJ, Rakheja D, Kovacs Z, et al. 2-Hydroxyglutarate detection by magnetic resonance spectroscopy in IDH-mutated patients with gliomas. Nat Med. (2012) 18:624-9. doi: $10.1038 / \mathrm{nm} .2682$

10. Andronesi OC, Kim GS, Gerstner E, Batchelor T, Tzika AA, Fantin VR, et al. Detection of 2-hydroxyglutarate in IDH-mutated glioma patients by in vivo spectral-editing and 2D correlation magnetic resonance spectroscopy. Sci Transl Med. (2012) 4:116ra4. doi: 10.1126/scitranslmed.3002693

11. Branzoli F, Di Stefano AL, Capelle L, Ottolenghi C, Valabrègue R, Deelchand DK, et al. Highly specific determination of IDH status using edited in vivo magnetic resonance spectroscopy. Neuro Oncol. (2018) 20:907-16. doi: 10.1093/neuonc/nox214

12. Bogner W, Gruber S, Trattnig S, Chmelik M. High-resolution mapping of human brain metabolites by free induction decay $1 \mathrm{H}$ MRSI at 7T. NMR Biomed. (2012) 25:873-82. doi: 10.1002/nbm.1805

13. Mekle R, Mlynárik V, Gambarota G, Hergt M, Krueger G, Gruetter R. MR spectroscopy of the human brain with enhanced signal intensity at ultrashort echo times on a clinical platform at 3T and 7T. Magn Reson Med. (2009) 61:1279-85. doi: 10.1002/mrm.21961

14. Choi C, Ganji S, Hulsey K, Madan A, Kovacs Z, Dimitrov I, et al. A comparative study of short-and long-TE $1 \mathrm{H}-\mathrm{MRS}$ at $3 \mathrm{~T}$ for in-vivo detection

\section{ACKNOWLEDGMENTS}

We thank the patients for participating in this study, and Sarah M. Jacobs for her assistance in performing the patients scan. We thank Vincent O. Boer for helping in sequence optimization.

\section{SUPPLEMENTARY MATERIAL}

The Supplementary Material for this article can be found online at: https://www.frontiersin.org/articles/10.3389/fneur. 2021.718423/full\#supplementary-material of 2-hydroxyglutarate in brain tumors. NMR Biomed. (2013) 26:1242-50. doi: $10.1002 / \mathrm{nbm} .2943$

15. Emir UE, Larkin SJ, de Pennington N, Voets N, Plaha P, Stacey $\mathrm{R}$, et al. Noninvasive quantification of 2-hydroxyglutarate in human gliomas with IDH1 and IDH2 mutations. Cancer Res. (2016) 76:43-9. doi: 10.1158/0008-5472.CAN-15-0934

16. Ganji SK, An Z, Tiwari V, McNeil S, Pinho MC, Pan E, et al. In vivo detection of 2-hydroxyglutarate in brain tumors by optimized pointresolved spectroscopy (PRESS) at 7T. Magn Reson Med. (2017) 77:936-44. doi: $10.1002 / \mathrm{mrm} .26190$

17. Berrington A, Voets NL, Larkin SJ, de Pennington N, Mccullagh J, Stacey $\mathrm{R}$, et al. A comparison of 2-hydroxyglutarate detection at 3 and $7 \mathrm{~T}$ with long-TE semi-LASER. NMR Biomed. (2018) 31:e3886. doi: 10.1002/nb m.3886

18. Andreychenko A, Boer VO, Arteaga de Castro CS, Luijten PR, Klomp DWJ. Efficient spectral editing at $7 \mathrm{~T}$ : GABA detection with MEGA-sLASER. Magn Reson Med. (2012) 68:1018-25. doi: 10.1002/mrm.24131

19. Tkáč I, Gruetter R. Methodology of 1 H NMR spectroscopy of the human brain at very high magnetic fields. Appl Magn Reson. (2005) 29:139-57. doi: $10.1007 / \mathrm{BF} 03166960$

20. Gruetter R. Automatic, localized in vivo adjustment of all firstand second-order shim coils. Magn Reson Med. (1993) 29:804-11. doi: 10.1002/mrm.1910290613

21. Hall EL, Stephenson MC, Price D, Morris PG. Methodology for improved detection of low concentration metabolites in MRS: optimised combination of signals from multi-element coil arrays. Neuroimage. (2014) 86:35-42. doi: 10.1016/j.neuroimage.2013.04.077

22. Simpson R, Devenyi GA, Jezzard P, Hennessy TJ, Near J. Advanced processing and simulation of MRS data using the FID appliance (FID-A)An open source, MATLAB-based toolkit. Magn Reson Med. (2017) 77:23-33. doi: $10.1002 / \mathrm{mrm} .26091$

23. Provencher SW. Estimation of metabolite concentrations from localizedin vivo proton NMR spectra. Magn Reson Med. (1993) 30:672-9. doi: $10.1002 / \mathrm{mrm} .1910300604$

24. Soher BJ, Semanchuk P, Todd D, Steinberg J, Young K. Proceedings of the 19th ISMRM and SMRT Annual Meeting and Exhibition, 7-13 May 2011 Montreal, QC (2011).

25. Cudalbu C, Behar KL, Bhattacharyya PK, Bogner W, Borbath T, de Graaf RA, et al. Contribution of macromolecules to brain $1 \mathrm{H} \mathrm{MR}$ spectra: experts' consensus recommendations. NMR Biomed. (2021) 34:e4393. doi: 10.1002/NBM.4393

26. Juchem C, de Graaf RA. B0 magnetic field homogeneity and shimming for in vivo magnetic resonance spectroscopy. Anal Biochem. (2017) 529:17-29. doi: 10.1016/j.ab.2016.06.003

27. de Graaf RA. In Vivo NMR Spectroscopy. Chichester: John Wiley and Sons, Ltd. (2019).

28. Juchem C, Cudalbu C, Graaf RA, Gruetter R, Henning A, Hetherington $\mathrm{HP}$, et al. B o shimming for in vivo magnetic resonance spectroscopy: experts' consensus recommendations. NMR Biomed. (2020) 34:e4350. doi: $10.1002 / \mathrm{nbm} .4350$

29. Moser P, Hingerl L, Strasser B, PovaŽan M, Hangel G, Andronesi OC, et al. Whole-slice mapping of GABA and GABA + at $7 \mathrm{~T}$ via adiabatic 
MEGA-editing, real-time instability correction, and concentric circle readout. Neuroimage. (2019) 184:475-89. doi: 10.1016/j.neuroimage.2018.09.039

30. Branzoli F, Pontoizeau C, Tchara L, Luisa A, Stefano D, Kamoun A, et al. Cystathionine as a marker for $1 \mathrm{p} / 19 \mathrm{q}$ codeleted gliomas by in vivo magnetic resonance spectroscopy. Neuro Oncol. (2019) 21:765-74. doi: 10.1093/neuonc/noz031

31. Branzoli F, Marjańska M. Magnetic resonance spectroscopy of isocitrate dehydrogenase mutated gliomas: current knowledge on the neurochemical profile. Curr Opin Neurol. (2020) 33:413-21. doi: 10.1097/WCO.0000000000000833

32. Shen X, Voets NL, Larkin SJ, de Pennington N, Plaha P, Stacey R, et al. A noninvasive comparison study between human gliomas with IDH1 and IDH2 mutations by MR spectroscopy. Metabolites. (2019) 9:35. doi: $10.3390 /$ metabo9020035

33. Emir U, Voets NE, Larkin SE, De Pennington N, Plaha PE, Stacey RE, et al. Proceedings of the 29th ISMRM and SMRT Annual Meeting and Exhibition, Virtual Meeting, 15-20 May 2021. Vancouver, CA (2021).
Conflict of Interest: The authors declare that the research was conducted in the absence of any commercial or financial relationships that could be construed as a potential conflict of interest.

Publisher's Note: All claims expressed in this article are solely those of the authors and do not necessarily represent those of their affiliated organizations, or those of the publisher, the editors and the reviewers. Any product that may be evaluated in this article, or claim that may be made by its manufacturer, is not guaranteed or endorsed by the publisher.

Copyright $\odot 2021$ Shams, van der Kemp, Emir, Dankbaar, Snijders, de Vos, Klomp, Wijnen and Wiegers. This is an open-access article distributed under the terms of the Creative Commons Attribution License (CC BY). The use, distribution or reproduction in other forums is permitted, provided the original author(s) and the copyright owner(s) are credited and that the original publication in this journal is cited, in accordance with accepted academic practice. No use, distribution or reproduction is permitted which does not comply with these terms. 